\title{
Synthesis and characterization of various surfactants for stabilized CuO powder
}

\author{
Alina MATEI ${ }^{1, a}{ }^{*}$, Vasilica TUCUREANU ${ }^{1,2, b}$, Marian POPESCU ${ }^{1}$, \\ Cosmin ROMANITAN ${ }^{1,3}$, Bogdan BIŢA ${ }^{1,3}$, lleana CERNICA ${ }^{1}$ \\ ${ }^{1}$ National Institute for Research and Development in Microtehnologies IMT-Bucharest, Bucharest, \\ Romania \\ ${ }^{2}$ Department of Materials Science, Transilvania University of Brasov, Brasov, Romania \\ ${ }^{3}$ Faculty of Physics, University of Bucharest, Magurele, Romania

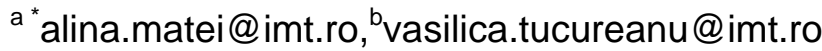

Keywords: Copper oxide, Nanoparticles, Surfactants, Chemical method

\begin{abstract}
In the present work, $\mathrm{CuO}$ nanoparticles were successfully prepared by the coprecipitation method using copper acetate $\left(\mathbf{C u}\left(\mathbf{C H}_{3} \mathbf{C O O}\right)_{2}\right)$ as a basic precursor, sodium hydroxide $(\mathrm{NaOH})$ as a precipitator material, sodium dodecyl sulfate (SDS) and cetyltrimethylammonium bromide (CTAB) as anionic and cationic surfactants, respectively. The synthesized powders samples were characterized by Fourier transform infrared spectrometry (FTIR), field emission scanning electron microscopy (FESEM) and X-ray diffraction (XRD). The investigation showed that the added types of surfactants have effects on the decrease of the crystallite size, on the $\mathrm{CuO}$ particles morphology, shape and uniform distribution as it is noticed in the XRD and SEM characterizations. Additionally, the FTIR spectra for all the powders samples showed the same $\mathrm{Cu}-\mathrm{O}$ stretching vibration mode which indicates the presence of a crystalline $\mathrm{CuO}$ monoclinic structure. The obtained results create premises for further advanced the applications of $\mathrm{CuO}$ powders in various domains.
\end{abstract}

\section{Introduction}

Over the years, the interests in developing nanoparticles metal oxides have considerably increased due to the necessity of obtaining materials with outstanding physical and chemical properties. Various methods of metal oxide synthesis have been know so far, researches continue to development a new approaches with a strict control over nanoparticles morphology, size and composition for several technological applications.

Copper oxide $(\mathrm{CuO})$, belonging to the nanomaterials class, which has attracted recent research because of its excellent properties, cost effectiveness and wide spectrum of practical applications (solar and electrochemical cells, gas sensors, field emitters, active catalyst and antimicrobial activity, etc.). Also, $\mathrm{CuO}$ as nanostructured oxide being classified as a $p$-type monoclinically structured semiconductor material with a direct band-gap value of $1.85 \mathrm{eV}$ presents a particular attention. This type of material has a special concern because it extends the use in a board range of applications, such as electronics and optoelectronics, catalysts, sensors and biosensors, chemical sensing devices, nanofluids and field emitters, desinfection, cosmetic pigments, antibacterial agent, etc. [1-4]. In order for this material to exhibit viable properties in the desired field of applicability, it is intended to establish its method of obtaining and its synthesis parameters; there

Content from this work may be used under the terms of the Creative Commons Attribution 3.0 license. Any further distribution of this work must maintain attribution to the author(s) and the title of the work, journal citation and DOI. Published under license by Materials Research Forum LLC. 
have been elaborated and known various physical and chemical methods so far, such as sol-gel, coprecipitation, hydrothermal synthesis, mechanical mixing, solid state reaction, thermal descomposition of precursors, microemulsion, microwave irraditiaon, physical vapor deposition, ablation, etc. [3, 5, 6].

From the bottom-up type methods, precipitation is a cheap one, with applicability on a broadscale, and which does not involve the addition of secondary reaction products [7-9]. Particularly, in order to obtain $\mathrm{CuO}$, the method involves precipitation of various soluble copper salts (nitrates, chlorides, sulphates and acetates) in aqueous solutions, followed by their thermal decomposition with oxide formation $[4,5,10]$.

Inorganic salts are mixed in an aqueous medium and under a rigorous $\mathrm{pH}$ control by using solutions of $\mathrm{NaOH}, \mathrm{KOH},\left(\mathrm{NH}_{4}\right)_{2} \mathrm{CO}_{3}$ or $\mathrm{NH}_{4} \mathrm{OH}$, finally the precipitates obtained are subject to characteristic thermal treatment. In the synthesis process the reaction parameters $(\mathrm{pH}$, rate of addition of reactants and speed of stirring, solution concentration, reaction temperature, etc.) have a determining role in the particle size, morphology and granulometry [11 - 13].

In the literature various thermal treatments at relatively high temperature (between $700{ }^{\circ} \mathrm{C}$ and $1100{ }^{\circ} \mathrm{C}$ ) are presented, leading to the phenomenon of agglomeration and increase of particles average size. To prevent the tendency of agglomeration and to favour the formation of nanostructured materials, the use surfactants have been proposed to be used. The use of different surfactants allows the improvement of particles structural, physico-chemical and morphological properties due to the electrostatic and stearic stabilizing mechanisms that reduce the solutions surface tension and improve the nanocrystalline material properties [6, $14-18]$.

Also, the addition of surfactants in the oxide materials precipitation process leads to increase nanoparticle stability and interaction between surfactant molecules and metal ions. The surfactants are chemical substances, focusing on the surface and solubilized materials with low affinity relative to each other. They have an asymmetrical molecular structure, consisting of a non-polar (hydrocarbon) and a polar (ionisable or non-ionisable) part. The role of surfactants is to provide an effective and efficient coating to induce electrostatic or stearic repulsions that can counterbalance van der Waals attractions [19-22].

The specialized literature shows research studies to improve the properties and formation of $\mathrm{CuO}$ nanoparticles by using various surfactants of the type of of oleic acid (OA), polyethylene glycol (PEG), cetyltrimethylammonium bromide (CTAB), hypochlorite dodecyl sulphate (SDS), polyvinylpyrrolidone (PVP), tetraoctylammonium bromide (TOAB), playing an important role in the synthesis process steps since the incipient phase. The reason for selecting the two types of surfactants (CTAB and SDS) in the experiments carried out in the present study is based on their remarkable effects on particle stability, size changing, morphology and the surface properties of the precipitated particles, but also because they have low price, can be found relatively easy on the market and have low toxicity [7, 14, 23 - 25].

This paper presents the study of obtaining $\mathrm{CuO}$ nanoparticles by the coprecipitation method, in the absence and in the presence of two types of surfactants, anionic (SDS) and cationic (CTAB). It is presented the effect of surfactants on $\mathrm{CuO}$ nanoparticles morphology, average size and crystalline structure. The morphological and structural properties are highlighted by using field emission scanning electron microscopy (FESEM), X-ray diffraction (XRD) and Fourier transform infrared spectrometry (FT-IR). 


\section{Experimental details}

For the synthesis of $\mathrm{CuO}$ powders: copper acetate monohydrate $\left[\mathrm{Cu}\left(\mathrm{CH}_{3} \mathrm{COO}\right)_{2}\right]$, sodium hydroxide $[\mathrm{NaOH}]$ and surfactants of type anionic $\left[\mathrm{C}_{12} \mathrm{H}_{25} \underline{\mathrm{NaO}}_{4} \mathrm{~S}\right.$, SDS 99\%] and $\left[\mathrm{C}_{19} \mathrm{H}_{42} \mathrm{BrN}\right.$, $\mathrm{CTAB}>99 \%$ ], were used as starting materials. All reagents were purchased from the company Sigma-Aldrich without any previous purification.

For the synthesis of $\mathrm{CuO}$ in the absence and in the presence of surfactants the stock solutions of $1 \mathrm{M}\left[\mathrm{Cu}\left(\mathrm{CH}_{3} \mathrm{COO}\right)_{2}\right]$ and $1 \mathrm{M}[\mathrm{NaOH}]$ were prepared, respectively. From the stock solution of acetate one part is taken and the $\mathrm{pH}$ is adjusted in basic medium by adding $\mathrm{NaOH}$ solution in dropwise until the formation of a greenish-blue precipitate was observed.

In the case of samples with surfactant, over the source of copper the afferent surfactants (SDS and CTAB concentration $0.1 \mathrm{M}$ ) was added and under continuous stirring the precipitating agent $(\mathrm{NaOH})$ was adding in dropwise until the precipitate formed and the $\mathrm{pH}$ was adjusted to about 10 11. After the precipitation formation, the stirring continues up to a temperature of $80{ }^{\circ} \mathrm{C}$. The introduction of SDS anionic surfactant induces a homogeneous nuclear process due to considerable size effect of counter-ions on the crystal facets. By adding CTAB as a cationic surfactant there is a complete ionization and cation formation in the tetrahedric structure, but it also determines a control of the growth rates of different faces of the $\mathrm{CuO}$ nanoparticles [14, 26].

For all synthesized samples the same synthetic conditions (time, temperature and $\mathrm{pH}$ ) were maintained. The precipitates thus formed are left in the rest position, then have been filtered under vacuum using a Buchner funnel, and following they were washed with a water-ethanol mixture for purification and removal of the secondary compounds. After washing, the samples were subjected to the drying step in the oven at a temperature of $80{ }^{\circ} \mathrm{C}$, preceded by calcination sintering at a temperature of $550{ }^{\circ} \mathrm{C}$ for 3 hours in normal atmosphere. Due to the sintering temperature of the dry samples changed colour from green to blue to black.

The functional groups and the chemical bonds of the synthesized oxide samples were analyzed by Fourier Transform Infrared spectrometry (Bruker Optics, Vertex 80V) using the $\mathrm{KBr}$ pellet method in the wavenumber range of $4000-400 \mathrm{~cm}^{-1}$ by averaging 64 scans. In the processing of all spectra, the bands attributable to the vibration mode of the $\mathrm{C}=\mathrm{O}$ bond in $\mathrm{CO}_{2}$ were extracted.

In order to investigate the morphology and particle size a Field Emission Scanning electron microscope (FE-SEM), obtained at an operating voltage at $10 \mathrm{kV}$ and a magnitude of $30000 \mathrm{x}$ has been used.

$\mathrm{X}$-ray diffraction measurements of the synthesized $\mathrm{CuO}$ particles were recorded using a Rigaku Smartlab diffractometer with the radiation $\mathrm{CuKa}=1.540593 \AA$, indicating the limit of variation for the current between $150 \mathrm{~mA}$ and $190 \mathrm{~mA}$. Data were collected at a scan rate of $12^{\circ} / \mathrm{min}$. in the range $20=20-95^{\circ}$.

\section{Results and Discussion}

\section{FTIR Spectra}

The FTIR spectra of the different samples are presented as a comparison between $\mathrm{CuO}$ powders obtained in the absence of surfactants (Figure 1a) and in the presence of surfactants (Fig. 1b and c).

For all samples treated at $550{ }^{\circ} \mathrm{C}$, bands attributed to both the vibration mode of the $\mathrm{Cu}-\mathrm{O}$ linkages in the precursor and to the surfactants vibration mode can be seen, thus suggesting the binding of surfactants to particle surface. Bands centred at wavelengths less than $600 \mathrm{~cm}^{-1}$, can be attributed to the vibration mode of $\mathrm{Cu}-\mathrm{O}$ bond from samples, indicating a temperature and time sufficient for total synthesis to obtain $\mathrm{CuO}$. 
Thus, bands that can be attributed to the $\mathrm{Cu}-\mathrm{O}$ bond at: a) 599, 511 and $430 \mathrm{~cm}^{-1}$ for the sample of $\mathrm{CuO}$ obtained in the absence of surfactants; b) 590, 488 and $423 \mathrm{~cm}^{-1}$ for the sample of CuO obtained in the presence of anionic surfactant (SDS); c) 608, 495 and $411 \mathrm{~cm}^{-1}$ for the sample of $\mathrm{CuO}$ obtained in the presence of the cationic surfactant (CTAB), confirming the formation of a $\mathrm{CuO}$ monoclinic crystalline structure.

In the case of $\mathrm{CuO}$ samples in the presence of surfactants (Fig. 1b and c) is observed the spectral bands centred in the region of $3000-800 \mathrm{~cm}^{-1}$ which can be attributed to the type of surfactant used. The FTIR spectra for the two samples presents the intense bands at $2920 \mathrm{~cm}^{-1}$, $2855 \mathrm{~cm}^{-1}$ and $1405 \mathrm{~cm}^{-1}$ for the sample with anionic surfactant and $2917 \mathrm{~cm}^{-1}, 2852 \mathrm{~cm}^{-1}$ and $1415 \mathrm{~cm}^{-1}$ respectively for the sample with cationic surfactant which may be due to vibration of the $\mathrm{C}-\mathrm{H}$ from organic materials. Also, the peaks from $1700-800 \mathrm{~cm}^{-1}$ may be assigned to specific bonds from surfactants. Based on the appearance of bands corresponding to the surfactants used in synthesized samples, reveals the interaction and binding of the surfactant molecules on the surface of $\mathrm{CuO}$ particles.

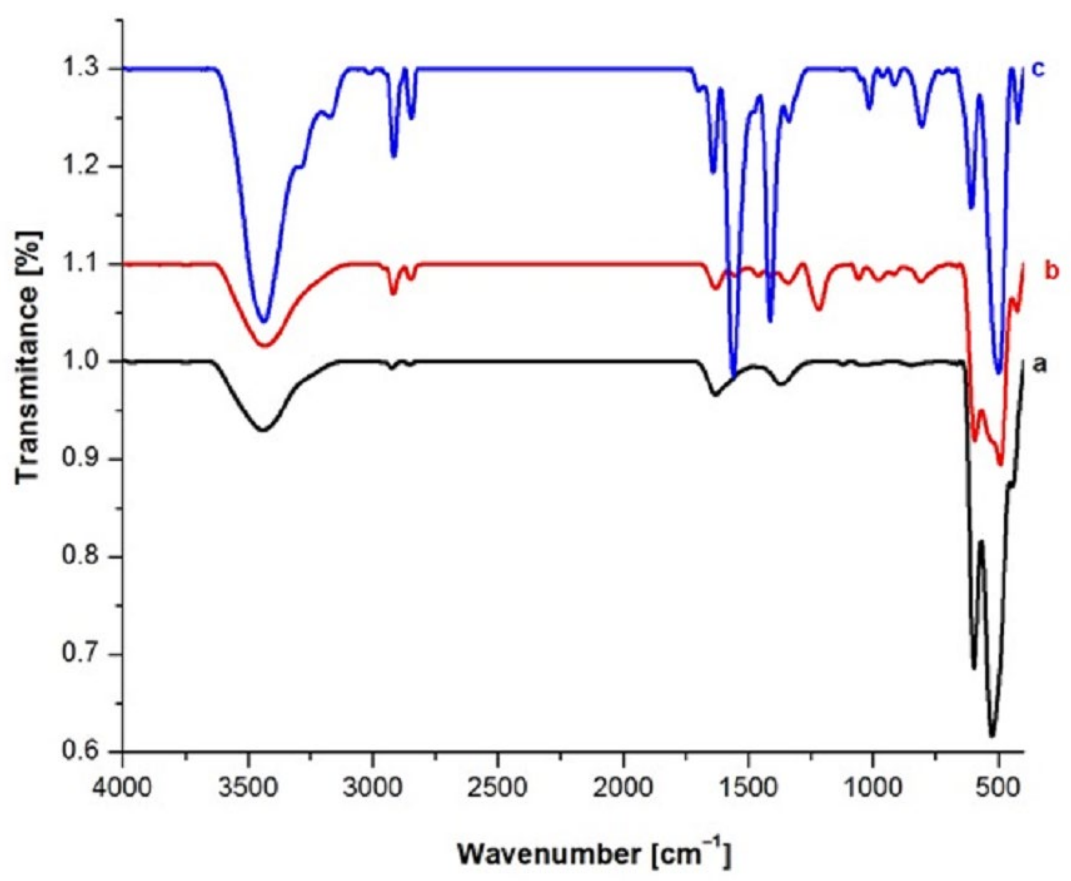

Fig. 1. FTIR spectra of $\mathrm{CuO}$ powders in the absence of surfactant (a) and in the presence of surfactants (b), SDS, (c) CTAB.

\section{Morphological Analysis}

Fig. 2 shows micrographs of $\mathrm{CuO}$ samples in the absence and the presence of surfactants. Analysis of samples by SEM was carried out for the correct estimation of their morphology, nanoparticle size, nanostructure formation and the agglomeration degree.

The $\mathrm{CuO}$ sample synthesized in the absence of surfactants (Fig. 2a) revealed agglomeration of particles, with dimensions in the range $40-70 \mathrm{~nm}$, showing an polyhedral shape with rounded edges for defining the particles shape. The use of different types of surfactants has been derived from the necessity to control the size and morphology of the nanostructures, as well as to reduce the agglomeration tendency of particles [14,23]. 
Thus, in the case of the $\mathrm{CuO}$ samples with anionic surfactant - SDS (Fig. 2b), from SEM micrograph we observed a slight decrease of the tendency of agglomeration concomitantly with particle size reduction, but the particle size diminishes, the particle size being estimated between 20 and $60 \mathrm{~nm}$. As a result of this observation, the necessity to find another surfactant in order to decrease furthermore the tendency of agglomeration arises.

The micrograph of $\mathrm{CuO}$ with surfactant cation - CTAB sample (Fig. 2b) shows the formation of particles with a well-defined shape, polyhedral with rounded edges, but with larger particle size due to the type of surfactant used and the high sintering temperature. This increase of particles size can be determined by the global tension forces that minimize free surface energy by controlling particle interaction, nucleation and particle size modification by keeping the particles at a nanometric level. It is well known that the speed of the cationic surfactants adsorption is rapid, indicating that the adsorption is faster compared with anionic surfactants, showing that the stabilization and the properties of the nanoparticules differ from the other surfactants.
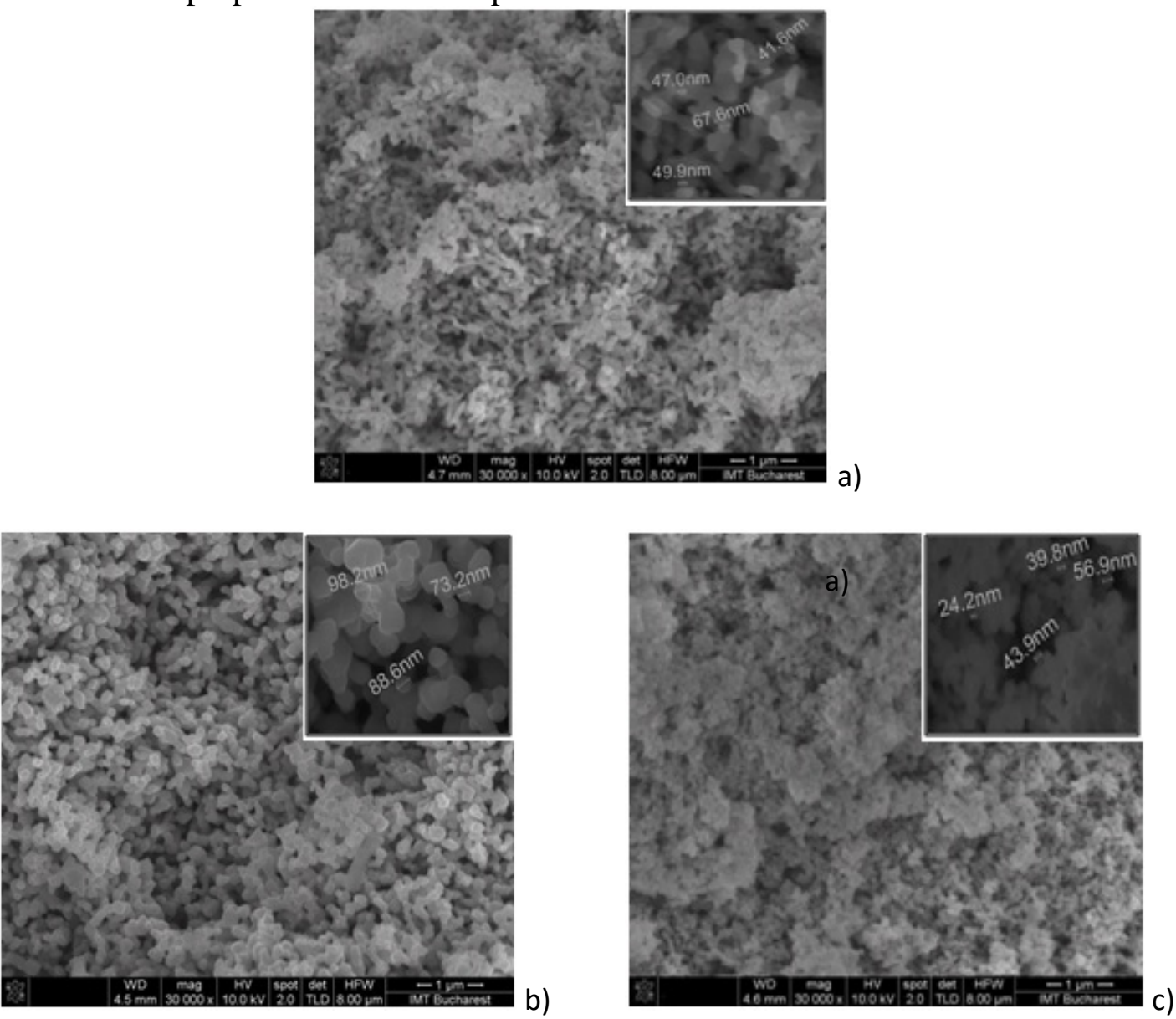

Fig. 2. SEM micrograph of $\mathrm{CuO}$ powder in the absence of surfactant (a) and in the presence of surfactants (b), SDS, (c) CTAB.

\section{XRD Analysis}

XRD diffraction spectra for $\mathrm{CuO}$ powders in the absence and in the presence of surfactants are highlighted in Fig. $3(\mathrm{a}-\mathrm{c})$. By using this characterization method the crystallite mean size, crystallographic structure and lattice parameters are estimate. In all three samples the diffraction 
peaks were indexed in accordance with the standard data of the No 00-101-1194, being in good accordance with the existing research literature [5,25,27].

From the analyzed samples it was found that the XRD diffractograms are similar and typical with the $\mathrm{CuO}$ single phase monoclinic structure, the surfactants do not influence the samples crystalline structure and no other intermediate phases characteristic of them have been observed. Also, the characteristic diffraction peaks centered at about $20(\mathrm{hkl})=32^{\circ}, 35^{\circ}, 38^{\circ}, 48^{\circ}, 53^{\circ}, 58^{\circ}$, $61^{\circ}, 68^{\circ}, 72^{\circ}, 75^{\circ}$ are shown, corresponding to the crystalline planes characteristic of $\mathrm{CuO}$ with Miller indices at (110), (002), (111), ( $\overline{202}),(020),(202),(\overline{113}),(220),(311),(004)$.

Depending on the sample type, the two main peaks of the crystalline planes with indices (002) and (111) can be found at $20=35,474^{\circ}$ and 38,714 ${ }^{\circ}$ for the $\mathrm{CuO}$ sample in the absence of surfactants, and at $2 \theta=35,426^{\circ}$ and $38,617^{\circ}$ for $\mathrm{CuO}$ samples in the presence of anionic surfactant (SDS) and at $2 \theta=35,377^{\circ}$ and $38,665^{\circ}$ respectively for $\mathrm{CuO}$ samples in the presence of CTAB. As a result of the use of surfactants, changes in the average size of crystallites and lattice parameters can be observed. The details of these parameters and the variation of the size of the crystals are shown in Table 1.

Calculation of the average crystallite size for all three samples was based on the Debye Scherer's formula (1):

$$
D=K \lambda /(\beta \cos \theta)
$$

Where: $K$ is the shape coefficient (0.94), $\lambda$ is the radiation wavelength (1.54 A), $\beta$ is the peak FWHM (full width half maximum width in radians), and $\theta$ is the Bragg diffraction angle obtained from $2 \theta$ value corresponding to maximum intensity peak (in radians).

Based on this formula the results indicate that the average size of crystallites varied from 16.7 $\mathrm{nm}$ for $\mathrm{CuO}$ and $12.5 \mathrm{~nm}$ for $\mathrm{CuO}$ in the presence of SDS and $20.02 \mathrm{~nm}$ for $\mathrm{CuO}$ in the presence of CTAB respectively, thus it was found that depending on the type of surfactant used, a control is performed on the degree of crystallites growth.

Table 1. Crystallite sizes, lattice parameters and range particle size of $\mathrm{CuO}$ powders in the absence and in the presence of surfactants.

\begin{tabular}{|c|c|c|c|c|c|}
\hline \multirow{2}{*}{\multicolumn{2}{|c|}{$\begin{array}{l}\text { Sample } \\
\text { number }\end{array}$}} & \multirow{3}{*}{$\begin{array}{c}\text { Crystallite } \\
\text { sizes (nm) } \\
16.7\end{array}$} & \multicolumn{2}{|c|}{ Lattice parameters } & \multirow{3}{*}{$\begin{array}{c}\begin{array}{c}\text { Range } \\
\text { particle sizes } \\
\text { (nm) }\end{array} \\
50\end{array}$} \\
\hline & & & \multirow{2}{*}{$\begin{array}{c}\text { a (A) } \\
4.6859\end{array}$} & \multirow{2}{*}{$\begin{array}{c}\text { c (A) } \\
5.1327\end{array}$} & \\
\hline 1 & $\mathrm{CuO}$ & & & & \\
\hline 2 & $\mathrm{CuO}$ in the presence of $S D S$ & 12.5 & 4.6857 & 5.1320 & 40 \\
\hline 3 & $\mathrm{CUO}$ in the presence of $C T A B$ & 20.02 & 4.6923 & 5.1394 & 70 \\
\hline
\end{tabular}




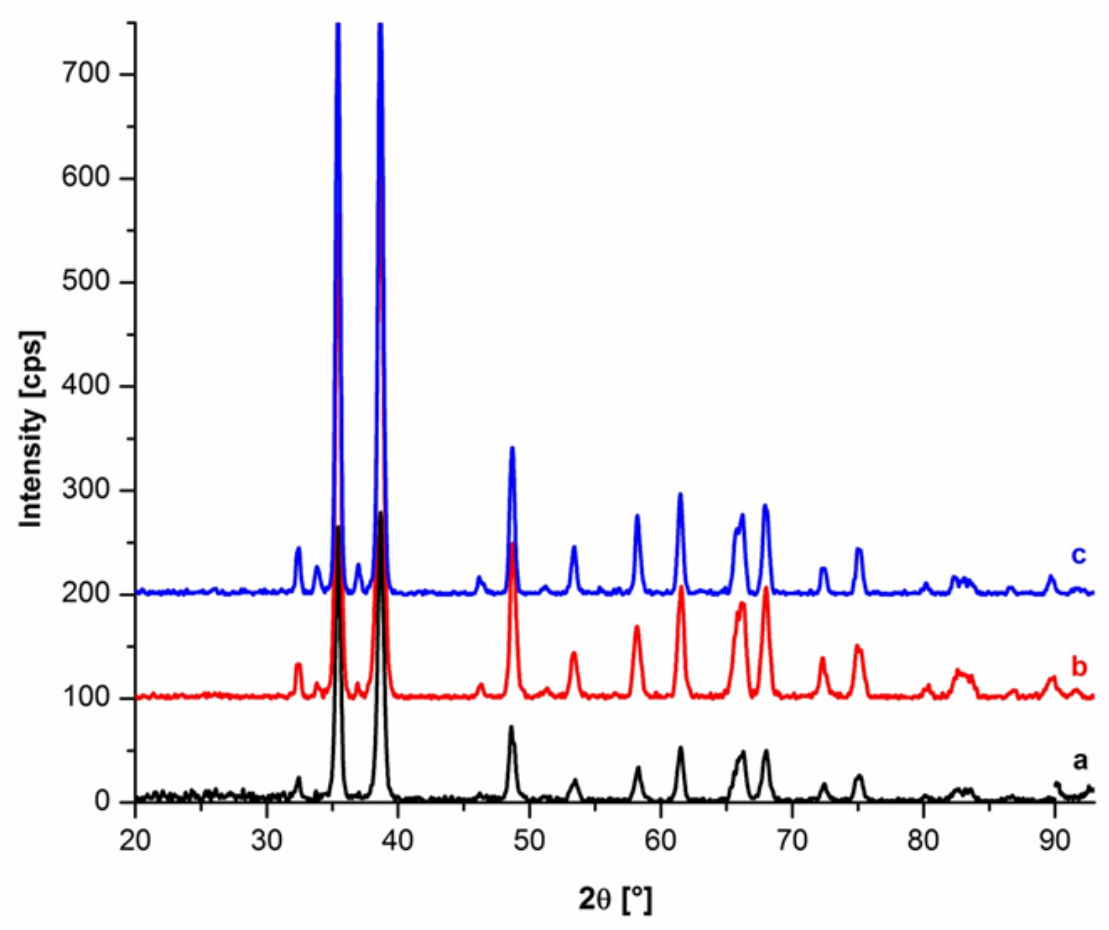

Fig. 3. XRD pattern of $\mathrm{CuO}$ powder in the absence of surfactant (a) and in the presence of surfactants (b), SDS, (c) CTAB.

\section{Summary}

In the present study, $\mathrm{CuO}$ nanoparticles were synthesized by the chemical co-precipitation method, in the absence and in the presence of two types of surfactants (SDS and CTAB), sintered at $550^{\circ} \mathrm{C}$. Structural analysis clearly indicates the formation of a single crystalline phase in all synthesized samples.

FTIR spectra reveal bands centered at wavelengths less than $600 \mathrm{~cm}^{-1}$ characteristic of the Cu$\mathrm{O}$ metal bond. Also, the presence of peaks in the region $3000-800 \mathrm{~cm}^{-1}$ indicates the binding of surfactants to the surface of the particles without affecting the particle crystalline structure, confirmed by XRD.

The XRD analysis indicates that regardless of the type of surfactant used, the powders synthesized had a $\mathrm{CuO}$ single phase monoclinic structure.

SEM images indicate that the sample obtained in the absence of surfactants shows an agglomeration tendency with dimensions ranging from 40-70 $\mathrm{nm}$.

In the case of samples synthesized depending on the type of surfactant, it is found that there is a control over the morphology and particle sizes ranging between $20-80 \mathrm{~nm}$ and reduces the agglomeration of the $\mathrm{CuO}$ particles.

The results showed that function of the type of surfactant used an improvement in the dispersion of $\mathrm{CuO}$ nanoparticles, a control of the morphology and particle size was exerted. By optimizing the conditions of processing and the establishment of surfactants, this method may be accessible for the synthesis of the various types of oxide materials with effect in a wide range of domains. 


\section{Acknowledgments}

This work was supported by a grant of the Ministry of National Education and Scientific Research, RDI Program for Space Technology and Advanced Research-STAR, project number 639/2017. Also, this work was supported by National Basic Funding Programme TEHNOSPEC Project No. PN1632/2016.

\section{References}

[1] R. R. Sandupatla, P. Veerasomaiah, Synthesis, characterization and photoluminescence study of $\mathrm{CuO}$ nanoparticles using aqueous solution method, Int. J Nanomater. Biostruct. 6 (2016) 30-33.

[2] A. El-Trass, H. ElShamy, I. El-Mehasseb et al., $\mathrm{CuO}$ nanoparticles : synthesis, characterization, optical properties and interaction with amino acids, Appl. Surf. Sci. 258 (2012) 2997-3001. https://doi.org/10.1016/j.apsusc.2011.11.025

[3] E. Darezereshki, F. Bakhtiari, Synthesis and characterization of tenorite (CuO) nanoparticles from smelting furnace dust (SFD), J Min. Metall. Sect. B-Metall. 49 (2013) 21-26. https://doi.org/10.2298/JMMB120411033D

[4] A. Rahnama, M. Gharagozlou, Preparation and properties of semiconductor CuO nanoparticles via a simple precipitation method at different reaction temperatures, Opt. Quant. Electron. 44 (2012) 313-322. https://doi.org/10.1007/s11082-011-9540-1

[5] S.J. Davarpanah, R. Karimian, V. Goodarzi et al., Synthesis of Copper (II) Oxide (CuO) nanoparticles and its application as gas sensor, J Appl. Biotechnol. Rep. 2 (2015) 329-332.

[6] M.E. Grigore, E.R. Biscu, A.M. Holban, et al., Methods of synthesis, properties and biomedical applications of $\mathrm{CuO}$ nanoparticles, Pharmaceuticals 9 (2016) 1-14. https://doi.org/10.3390/ph9040075

[7] J. Singh, G. Kaur, M. Rawat, A brief review on synthesis and characterization of copper oxide nanoparticles and its applications, J Bioelectron. Nanotechnol. 1 (2016) 1-9.

[8] A. Matei, V. Tucureanu, L. Dumitrescu, Aspects regarding synthesis and applications of ZnO. Bull Transilv Univ Brasov, Ser.I: Eng. Sci. 7 (2014) 45-52.

[9] T. Iqbal, A. Hassan, S. Ghazal, Synthesis of iron oxide, cobalt oxide and silver nanoparticles by different techniques: a review, Int. J Sci. Eng. Res. 7 (2016) 1178-1221.

[10] K. Phiwdang, S. Suphankij, W. Mekprasart, Synthesis of CuO nanoparticles by precipitation method using different precursors, Energy Procedia. 34 (2013) 740-745. https://doi.org/10.1016/j.egypro.2013.06.808

[11] M.F. Romadhan, N.E. Suyatma, F.M. Taqi, Synthesis of ZnO nanoparticles by precipitation method with their antibacterial effect, Indones. J Chem. 16 (2016) 117-123. https://doi.org/10.22146/ijc.21153

[12] D. Raoufi, Synthesis and microstructural properties of $\mathrm{ZnO}$ nanoparticles prepared by $\begin{array}{lllll}\text { precipitation method, } & \text { Renew. } & \text { Energy. } & 50 & \text { (2014) 937. }\end{array}$ https://doi.org/10.1016/j.renene.2012.08.076

[13] M.C. Mascolo, Y. Pei, T.A. Ring, Nanoparticles in a large $\mathrm{pH}$ window with different bases, Materials 6 (2013) 5549-5567. https://doi.org/10.3390/ma6125549 
[14] H. Siddiqui, M.S. Qureshi, F.Z. Haque, Optik surfactant assisted wet chemical synthesis of copper oxide (CuO) nanostructures and their spectroscopic analysis, Optik 127 (2016) 2740-2747. https://doi.org/10.1016/j.ijleo.2015.11.220

[15] A.K. Arora, Synthesis of nanosized CuO particles: a simple and effective method, Int. J Chem. Sci. 11 (2013) 1270-1276.

[16] T.H. Tran, V.T. Nguyen, Copper oxide nanomaterials prepared by solution methods, some properties, and potential applications:a brief review, Int. Sch. Res. Notices 2014 (2014) 1-14. https://doi.org/10.1155/2014/856592

[17] R. Eview, Methods of preparation of nanoparticles- a review, Int. J Adv. Eng. Techol. 7 (2015) 1806-1811.

[18] P. Derakhshi, R. Lotfi, Synthesis and surfactant effect on structural analysis of nickel doped cobalt ferrite nanoparticles by c-precipitation method, J Appl. Chem. Res. 6 (2012) 60-65.

[19] S.K. Mehta, S. Kumar, S. Chaudhary et al., Evolution of ZnS nanoparticles via facile CTAB aqueous micellar solution route: a study on controlling parameters, Nanoscale Res. Lett. 4 (2009) 17-28. https://doi.org/10.1007/s11671-008-9196-3

[20] L.P. Singh, S.K. Bhattacharyya, G. Mishra, S. Ahalawat, Functional role of cationic surfactant to control the nano size of silica powder, Appl. Nanosci. 1 (2011) 117-122. https://doi.org/10.1007/s13204-011-0016-1

[21] M. Manisha, P. Muthuprasanna, Surya prabha K et al., Basics and potential applications of surfactants - a review, Int. J Pharm. Tech. Res. 1 (2009) 1354-1365.

[22] R. Azarmi, A. Ashjaran, Type and application of some common surfactants, J Chem. Pharmacal. Res. 7 (2015) 632-640.

[23] M. Saterlie, H. Sahin, B. Kavlicoglu et al., Particle size effects in the thermal conductivity enhancement of copper-based nanofluids, Nanoscale Res. Lett. 6 (2011) 1-7. https://doi.org/10.1186/1556-276X-6-217

[24] A. Ananth, S. Dharaneedharan, M.S. Heo et al., Copper oxide nanomaterials: synthesis, characterization and structure-specific antibacterial performance, Chem. Eng. J. 262 (2015) 179188. https://doi.org/10.1016/j.cej.2014.09.083

[25] X. Wang, J. Yang, L. Shi et al., Surfactant-free synthesis of CuO with controllable morphologies and enhanced photocatalytic property, Nanoscale Res. Lett. 11 (2016) 125. https://doi.org/10.1016/j.cej.2014.09.083

[26] L.S. Cavalcante, J.C. Sczancoski, M.S. Li et al., Aspects method: growth process and photoluminescence properties, Colloids Surf A: Physicochem Eng. Asp. 396 (2012) 346-351. https://doi.org/10.1016/j.colsurfa.2011.12.021

[27] B. Shaabani, E. Alizadeh-Gheshlaghi, E. Azizian-Kalandaragh et al., Preparation of $\mathrm{CuO}$ nanopowders and their catalytic activity in photodegradation of Rhodamine-B, Adv. Powder Technol. 25 (2014) 1043-1052. https://doi.org/10.1016/j.apt.2014.02.005 